\title{
Cardiovascular magnetic resonance imaging: emerging techniques and applications
}

\author{
Amrit Chowdhary ${ }^{1}$, Pankaj Garg ${ }^{2}$, Arka Das $^{1}$, Muhummad Sohaib Nazir ${ }^{3}$, Sven Plein ${ }^{1,3}$, \\ Christopher I Kelly \\ ${ }^{1}$ Leeds Institute of Cardiovascular and Metabolic Medicine, University of Leeds, Leeds, UK \\ ${ }^{2}$ Cardiovascular and Metabolic Medicine Group, University of East Anglia, Norwich, UK \\ ${ }^{3}$ School of Biomedical Engineering and Imaging Sciences, King's College London, London, UK
}

\begin{abstract}
This review gives examples of emerging cardiovascular magnetic resonance (CMR) techniques and applications that have the potential to transition from research to clinical application in the near future. Four-dimensional flow CMR (4D-flow CMR) allows time-resolved three-directional, three-dimensional (3D) velocity-encoded phase-contrast imaging for 3D visualisation and quantification of valvular or intracavity flow. Acquisition times of under $10 \mathrm{~min}$ are achievable for a whole heart multidirectional data set and commercial software packages are now available for data analysis, making 4D-flow CMR feasible for inclusion in clinical imaging protocols. Diffusion tensor imaging (DTI) is based on the measurement of molecular water diffusion and uses contrasting behaviour in the presence and absence of boundaries to infer tissue structure. Cardiac DTI is capable of non-invasively phenotyping the 3D micro-architecture within a few minutes, facilitating transition of the method to clinical protocols. Hybrid positron emission tomographymagnetic resonance (PET-MR) provides quantitative PET measures of biological and pathological processes of the heart combined with anatomical, morphological and functional CMR imaging. Cardiac PET-MR offers opportunities in ischaemic, inflammatory and infiltrative heart disease.
\end{abstract}

\section{Introduction}

The indications for cardiovascular magnetic resonance (CMR) in international clinical practice guidelines continue to increase. CMR methods used in current clinical routine include anatomical imaging, cine imaging, flow velocity-encoded acquisition, angiography, dynamic and delayed contrast enhancement. Other CMR methods continue to transition into

\footnotetext{
Correspondence to: Sven Plein.

Correspondence to: Prof Sven Plein, Leeds, Yorkshire, UK; s.plein@leeds.ac.uk.

Twitter Amrit Chowdhary @DrAChowdhary, Muhummad Sohaib Nazir@ sohaibnazir and Sven Plein @ Sven Plein

Contributors All authors have contributed to literature search and writing of the manuscript.

Competing interests None declared.

Patient and public involvement Patients and/or the public were not involved in the design, or conduct, or reporting, or dissemination plans of this research.

Patient consent for publication Not required.

Provenance and peer review Commissioned; externally peer reviewed.
} 
clinical routine, such as parametric mapping, which has recently been included in guidelines for the diagnosis of myocarditis. This review gives an overview of some other CMR methods that have the potential to move from research to clinical application in the near future.

\section{Four-Dimensional Flow CMR}

Blood flow in the heart is complex, dynamic and has a three-dimensional (3D) profile. ${ }^{1}$ Non-invasive imaging techniques, such as Doppler echocardiography and two-dimensional (2D) cine phase-contrast (PC) CMR are used in clinical routine to measure blood flow in the heart and vasculature, however, these methods cannot fully assess the complex, 3D and multidirectional nature of intracardiac blood flow.

Four-dimensional flow CMR (4D-flow CMR) is an emerging technology which allows timeresolved three-directional, 3D velocity-encoded $\mathrm{PC}$ imaging for 3D visualisation and quantification of valvular or intracavity flow ${ }^{2}$ (figure 1 ). Whole-heart 4D-flow CMR can be performed in under 10 min using retrospective ECG gating and free-breathing acquisition ${ }^{34}$ and several commercial solutions for data analysis are now available. These developments allow the inclusion of 4D-flow CMR in clinical protocols. The potential clinical applications of 4D-flow include qualitative assessment of complex flow patterns and vortices as well as quantitative measurement of valve regurgitation and kinetic energy (KE) (Table 1).

\section{Visualisation of complex intracavity flow}

4D-flow CMR data can be co-registered with cine images and displayed as two-directional vectors and colour coded with velocity information. Such an overlay allows novel insight into normal and abnormal flow patterns associated with cardiac disease. For example, an eccentric mitral regurgitation jet may be swirling in the entire left atrium and hence may not be limited to the defined backward flow seen by Doppler echocardiography. In addition, using 4D-flow CMR one can visualise flow accelerations through stenotic valvular or vascular lesions. This can provide complementary clinical information in challenging congenital heart disease cases where haemodynamic effects of anatomical abnormalities are not always clear. The visualisation of two-directional vectors may also enhance detection of septal defects, flow acceleration in outflow tracts and valvular regurgitation.

\section{Vortex imaging}

4D-flow CMR can help understand flow behaviour inside the cardiac chambers. One of the main mechanisms of intracardiac flow is vortex behaviour. Vortices can form in all chambers of the heart and in the major vessels. They can represent either optimal physiological flow ${ }^{5}$ or pathological flow due to dilatation ${ }^{6}$ or raised pressures. ${ }^{7}$ For example, vortical blood flow in the main pulmonary artery $>14.3 \%$ of the cardiac interval corresponds to pulmonary hypertension with $97 \%$ sensitivity and $96 \%$ specificity. Similarly, in aortic root dilation, more helical flows with vortices can be identified with either 2D or 3D flow visualisation. Several quantification tools are under development to inform clinical management in aortopathy. 


\section{Quantification of 4D-flow}

4D-flow CMR allows the measurement of several quantitative haemodynamic metrics. These include valvular flow, intracavity velocity or KE assessment, vortex quantification, blood flow component analysis and haemodynamic force quantification. ${ }^{8}$ According to a recent systematic review, the most clinically applicable technique is valvular flow quantification using retrospective valve tracking, ${ }^{8}$ while intracavity blood flow KE mapping shows promise. ${ }^{9}$

Retrospective valve tracking-Retrospective valve tracking using 4D-flow CMR allows quantification of flow through all four heart valves for the same averaged cardiac cycle, while factoring in valve motion by tracking the valve ${ }^{10}$ (figure 1 ). This method overcomes limitations that arise in conventional 2D CMR or echocardiographic flow imaging from physiological variation, throughplane motion and selection of the best imaging plane with more precise calculation of flows and intracardiac shunts. In this context, 4Dflow CMR can be considered to be the non-invasive 'gold-standard' for intracardiac flow quantification. ${ }^{11}$ In patients with limited echocardiographic views, 4D-flow CMR derived mitral inflow provides an alternative to assess peak $\mathrm{E}$ and A velocities for left ventricular (LV) diastolic function assessment. In addition, it can be applied to directly quantify regurgitant volumes by prescribing an analysis plane in the 4D volume data set that is exactly perpendicular to the regurgitant jet. ${ }^{12}$ Automated valve tracking procedures are becoming available which reduce postprocessing time significantly. ${ }^{13}$

Quantification of intracavity kinetic energy-Intracavity LV blood flow can be quantified for the complete cardiac cycle by $4 \mathrm{D}$-flow CMR. This is achieved by superimposing endocardial contours defined from cine CMR images onto 4D-flow data. This process allows to quantify LV blood flow KE (figure 2). The KE of the blood represents a fundamental component of work performed by the heart which results in the movement of the blood. KE assessment factors in velocity in all the three directions (x, y and Z) per voxel. For each volumetric element (voxel), the formula for computing KE is $\mathrm{KE}=1 / 2$ pblood. $\mathrm{V}_{\text {voxel }} \cdot \mathrm{v}^{2}$ where $\rho_{\text {blood }}$ is the density of blood $\left(1.06 \mathrm{~g} / \mathrm{cm}^{3}\right), \mathrm{V}_{\text {voxel }}$ is the voxel volume and $\mathrm{v}$ the velocity magnitude. For each cardiac phase, the total KE within the LV is obtained by summation of the KE of every voxel. The analysis of KE is largely automated and a key strength of this technique is its excellent intraobserver/interobserver agreement (coefficient of variation $=6 \% \pm 1 \%$ ) and almost immediate haemodynamic assessment without requiring additional segmentations to those acquired in routine practice. ${ }^{8}$ Importantly, as it factors in the complete intracavity flow, LV blood flow KE is a finer marker of diastolic function than standard diastolic parameters. ${ }^{8}$ Also, other studies have shown how it independently predicts adverse remodelling postmyocardial infarction (MI) ${ }^{14}$ and LV thrombus formation. ${ }^{15}$ Therefore, this emerging tool for haemodynamic assessment holds great promise for phenotyping cardiac haemodynamics, informing diagnosis and potentially predicting disease course. Future trials are needed to evaluate its value in informing treatment outcomes in cardiovascular diseases. 


\section{Clinical potential of 4D-flow}

Four-dimensional flow imaging with CMR has reached a level of technical maturity that allows it to migrate from research to clinical application. Acquisition times of under $10 \mathrm{~min}$ are achievable for a whole heart multidirectional data set and commercial software packages are available for data analysis. However, standardisation and validation of acquisition and analysis protocols and large-scale evidence is needed to define its future role. Postprocessing remains time-consuming and automisation of at least some analysis steps will be essential to facilitate clinical translation. The first clinical applications of 4D-flow CMR are likely to be in assessment of aortic and complex congenital heart disease (table 1).

\section{Diffusion Tensor Imaging}

Diffusion tensor imaging (DTI) is already in clinical use outside of the heart and has the unique ability to characterise microstructural tissue changes. Application of this method for cardiac imaging has been challenging, but in recent years cardiac DTI methods have shown clinical potential.

DTI is based on the measurement of molecular water diffusion and uses contrasting behaviour in the presence and absence of boundaries to infer tissue structure (table 1). Where there are no boundaries, water molecules diffuse freely, while in the presence of physiological barriers (such as cell membranes, capillaries and extracellular matrix) this free diffusion is restricted. DTI can be used to probe the primary directions and magnitudes of diffusion. ${ }^{16-18}$ Cardiac DTI is capable of non-invasively phenotyping the 3D microarchitecture of the heart. ${ }^{19}$ In addition, by acquiring data in multiple phases of the cardiac cycle, DTI can provide dynamic information and improve understanding of myocardial laminar structure and its complex inter-play with functional mechanics.

For DTI reconstruction, diffusion is measured in at least six different directions, which enables the mathematical description of diffusion using tensor notation; pictorially, tensors can be described using ellipsoids whose primary axis coincides with the primary diffusion direction. ${ }^{20}$ Based on the derived tensor representation, it is possible to infer in each voxel a number of different tissue characteristics. The molecular diffusion rate or mean diffusivity (MD) is a measure of how freely water can diffuse in the myocardium, while the degree of diffusion restriction in different directions is measured using fractional anisotropy (FA) (figure 3). The underlying tissue structure orientation is described using the eigenvectors of the diffusion tensor. These lead to commonly reported measures such as the helix angle (HA) and second eigenvector angle (E2A) (figure 4). The HA is a measure of the orientation of cardiomyocytes, which changes transmurally from left-handed at the epicardium, to circumferential in the midwall and right-handed at the endocardium. HA maps depict the average orientation of cardiomyocytes on a voxel-wise basis. E2A describes the angulation of laminar sheetlets in the myocardial wall (the angle between the cross-myocyte eigenvector E2 and radial direction), and values can also be plotted on a map to depict the local contractile state of the myocardium.

DTI has been shown to be reproducible in studies involving both healthy and diseased cohorts. $^{20-22}$ Furthermore, in healthy volunteers regional heterogeneity of DTI parameters 
mentioned above have been demonstrated as well as the direct relationship between the E2A and HA gradients with LV mass and body surface area. ${ }^{23}$ An inverse relationship between E2A mobility and both age and ejection fraction of the LV has been described. ${ }^{24}$ These indices have been used in ex vivo and in vivo studies of healthy and diseased myocardium (figure 5).

\section{Myocardial infarction}

Following acute MI, myocardial necrosis and oedema lead to disruption of the myocardial micro-architecture in the infarct zone and in the peri-infarct tissue. DTI studies have demonstrated an increased MD and a decreased FA in the acute infarct zone when compared with remote myocardial segments. ${ }^{2526}$ Tractographic propagation angle (PA), which is a newer metric of cardiac myofibre architecture derived from DTI, has been used to delineate regions of infarct and shows good correlation with late gadolinium enhancement (LGE) imaging without the need for contrast agents. ${ }^{27}$ Gaining a better understanding of the microstructural effects of myocardial ischaemia and infarction using DTI should help elucidate the intricacies of the pathophysiological processes that lead to adverse LV remodelling.

\section{Hypertrophic cardiomyopathy}

Myocardial hypertrophy that is not solely explained by abnormal loading conditions and myofibre disarray are hallmarks of hypertrophic cardiomyopathy (HCM). Using DTI, the presence of myofibre disarray and dynamic myocardial microstructure alterations in the cardiac cycle have been demonstrated; while E2A and MD are elevated, FA is low in patients with HCM when compared with controls. ${ }^{2829}$ FA has also been studied as a marker of disarray, with the potential to identify patients at risk of ventricular arrhythmias and sudden cardiac death. ${ }^{29}$

\section{Dilated cardiomyopathy}

Ventricular chamber enlargement, systolic dysfunction and LV wall thinning are characteristic findings in dilated cardiomyopathy (DCM). A reduced systolic E2A, sheetlet mobility and FA, along with raised MD have been demonstrated in patients with DCM when compared with controls. ${ }^{3031}$ In contrast to healthy controls, the change in HA from diastole to systole was far less pronounced in DCM and the diastolic myofibre arrangement had a more longitudinal orientation. ${ }^{31}$ It is anticipated that an assessment of myocardial microstructure will provide a new tool for risk-stratification of patients with DCM.

\section{Congenital heart disease}

In congenital heart disease (CHD), significant derangement of myofibre orientation has been demonstrated in situs inversus totalis using DTI ${ }^{32}$ A predominance of longitudinal and oblique fibres in the systemic right ventricle hypothesised to be an adaptive response to systemic pressure and load has been demonstrated by DTI. ${ }^{33}$ DTI is expected to improve understanding of the complex effect of CHD on the myocardial microstructure with potential impact on clinical care. 


\section{Clinical potential of cardiac DTI}

Although at an early stage of its clinical development, cardiac DTI offers the unique potential of providing a non-invasive window into the microstructure of the heart, previously only accessible with invasive myocardial biopsy. Cardiac DTI images can now be acquired within a few minutes, making integration into clinical studies a realistic option. While data analysis remains time consuming and limited to specialist centres and consensus on the most robust DTI-derived measurements is pending, these current limitations are likely to be overcome within the next years. As for 4D-flow, standardisation of acquisition and analysis as well as faster, partly automated postprocessing will be required before the method transcends into clinical practice. Artificial intelligence will provide some of the necessary technical developments and the Society for Cardiovascular Magnetic Resonance is promoting standardisation of methods. The anticipated clinical use of cardiac DTI is in cardiomyopathies and congenital heart disease, but other applications are likely to emerge (table 1).

\section{Positron Emission Tomography-Magnetic Resonance}

Hybrid positron emission tomography-magnetic resonance (PET-MR) systems have recently been introduced and are finding clinical applications in many body systems, with cardiovascular indications beginning to emerge. Cardiac PET provides quantitative measures of biological and pathological processes of the heart, while CMR derives anatomical, morphological and functional imaging. The combined assessment of PET and CMR offers opportunities in ischaemic, inflammatory and infiltrative heart disease (table 1).

\section{Ischaemic heart disease}

Viability-PET-MR has potential to more accurately predict functional recovery than PET or CMR alone by providing an assessment of the metabolic activity of cells from ${ }^{18} \mathrm{~F}$ fluorodeoxyglucose $\left({ }^{18} \mathrm{~F}-\mathrm{FDG}\right)$, with tissue perfusion assessment by PET or CMR, scar imaging with LGE CMR and functional reserve with low-dose dobutamine CMR. This combined information may be particularly relevant in patients with intermediate scar extent of $25 \%-75 \%$ transmurality where the specificity of LGE CMR alone is low for prediction of functional recovery. In a small study of patients scheduled for coronary revascularisation, ${ }^{18}$ F-FDG PET-MR reclassified 19\% of non-assessable segments with intermediate extent of LGE. ${ }^{34}$ Another study demonstrated a poor correlation between hibernating myocardium defined by perfusion/viability mismatch with ${ }^{13} \mathrm{~N}$-Ammonia/ $/{ }^{18} \mathrm{~F}$-FDG PET and LGE with CMR, although did not use low-dose dobutamine stress CMR, which suggests that both modalities may confer synergism. ${ }^{35}$ Larger clinical studies and outcome data are required to determine the incremental prognostic benefit of PET-MR in hibernating myocardium.

Coronary imaging-PET-MR allows combined assessment of coronary anatomy and metabolism. There are emerging CMR methods to obtain high-resolution isotropic coronary images in short scan times. ${ }^{36}$ Using PET-MR, advanced reconstruction methods with nonrigid respiratory and cardiac deformation fields estimated from CMR can be used to motioncorrect CMR angiography and ${ }^{18} \mathrm{~F}-\mathrm{FDG}$ data. ${ }^{37} \mathrm{In}$ a pilot study, ${ }^{18} \mathrm{~F}$-fluoride was found to be a marker of high-risk vulnerable plaque localised by $\mathrm{CMR}^{38}$ and a recent study has 
demonstrated good agreement between quantitative PET-CT and PET-MR data. ${ }^{39}$ Future studies may obtain radiation-free coronary imaging alongside motion-corrected PET data to localise and identify metabolically active foci indicative of vulnerable coronary plaque prior to adverse cardiac events.

\section{Inflammatory heart disease}

Active inflammatory myocardial cells have greater glucose utilisation which makes ${ }^{18} \mathrm{~F}$ FDG attractive to evaluate inflammation. ${ }^{40}$ In a PET-MR study of patients with suspected myocarditis, PET and CMR provided complementary data and showed good agreement between ${ }^{18} \mathrm{~F}$-FDG and T2-weighted CMR and LGE. ${ }^{41}$ Emerging radiotracers such as ${ }^{68} \mathrm{GA}$ DOTATATE are sensitive to macrophage activity, ${ }^{42}$ which may be co-registered with areas of T2 mapping with CMR to detect early disease prior to development of fibrosis.

The diagnosis of cardiac sarcoidosis is challenging and both PET or CMR are recommended in current guidelines. ${ }^{43}$ Several small studies have confirmed the technical feasibility of hybrid PET-MR in suspected cardiac sarcoidosis. These have suggested different patterns of either ${ }^{18}$ F-FDG uptake or LGE or both, ${ }^{44}$ with sometimes poor agreement between the two methods, suggesting they track different pathology. ${ }^{45}$ Thus, PET-MR may provide complementary data with active metabolic inflammation from PET alongside fibrosis from CMR, which can lead to new classifications of the presence and activity of cardiac sarcoidosis $^{46}$ (figure 6).

Future PET-MR studies with novel inflammation tracers in patients with well-characterised phenotype of cardiac sarcoidosis may provide mechanistic insights into disease process, improve diagnosis and guide anti-inflammatory therapy.

\section{Infiltrative heart disease}

CMR has clinical utility for cardiac amyloidosis with $\mathrm{T} 1$ parametric mapping, extracellular volume and LGE. From a nuclear medicine perspective, ${ }^{99 \mathrm{~m}} \mathrm{Tc}-3,3$-diphosphono-1,2propanodicarboxylic acid bone scintigraphy can be used to accurately differentiate between AL and ATTR amyloid and provide semi-quantitative markers of amyloid burden. ${ }^{47}$ One PET-MR study demonstrated greater ${ }^{18} \mathrm{~F}$-fluoride uptake in areas of LGE in patients with ATTR amyloid compared with AL amyloid and healthy controls using PET-MR ${ }^{48}$ Other emerging PET tracers have been shown to detect cardiac amyloidosis such as ${ }^{18} \mathrm{~F}$ florbatepen ${ }^{49}$ and ${ }^{11} \mathrm{C}$-Pittsburgh compound $\mathrm{B},{ }^{50}$ with potential to provide absolute quantitative measures of amyloid burden. Future studies may investigate these tracers and co-register and motion-correct the PET data with CMR data to provide quantitative markers of amyloid burden, which may be used to monitor response to chemotherapeutic agents.

Cardiac masses-PET-MR has been used to investigate cardiac masses using ${ }^{18} \mathrm{~F}-\mathrm{FDG}$, although without parametric mapping or first-pass perfusion as part of the CMR protocol. ${ }^{41}$ The absolute clinical utility was limited, since there was a high diagnostic performance of standalone PET and CMR. However, PET-MR may provide additional information in cardiac masses that standalone imaging cannot fully characterise and potentially guide intracardiac biopsy. 


\section{Clinical potential of PET-MR}

Where available, cardiac PET-MR can already be integrated into clinical workflows when evidence for the individual use of PET and CMR is available (Table 1). As outlined above, the most likely clinical use is in inflammatory and infiltrative heart disease and in the assessment of cardiac masses. However, access to PET-MR is currently limited to a few specialist centres, with wider availability required for the method to become a common clinical tool. Developments of novel radiotracers, sequence development, attenuation correction, co-registration, motion correction and postprocessing with dedicated software for image fusion will further expand its clinical potential to gain mechanistic insight into disease, improve diagnoses, develop novel imaging biomarkers, guide therapeutics and provide risk stratification.

\section{Conclusions}

4D-flow, DTI and PET-MR are examples of the recent advances in CMR that are crossing the boundary from research to clinical practice. They promise better understanding of disease mechanisms and the potential for improved disease detection and risk stratification. Key challenges are acquisition time for complex methods and standardisation and, in particular in the case of PET-MR, limited access to the required hardware. As these challenges are overcome, it is likely that CMR methods in current development will become available for clinical use.

\section{Funding}

SP is funded by a British Heart Foundation Chair (CH/16/2/32089).

\section{References}

1. Taylor TW, Yamaguchi T. Flow patterns in three-dimensional left ventricular systolic and diastolic flows determined from computational fluid dynamics. Biorheology. 1995; 32:61-71. [PubMed: 7548861]

2. van der Geest RJ, Garg P. Advanced analysis techniques for Intra-cardiac flow evaluation from 4D flow MRI. Curr Radiol Rep. 2016; 4:38. [PubMed: 27390626]

3. Garg P, Westenberg JJM, van den Boogaard PJ, et al. Comparison of fast acquisition strategies in whole-heart four-dimensional flow cardiac Mr: two-center, 1.5 Tesla, phantom and in vivo validation study. J Magn Reson Imaging. 2018; 47:272-81. [PubMed: 28470915]

4. Bock J, Töger J, Bidhult S, et al. Validation and reproducibility of cardiovascular 4D-flow MRI from two vendors using $2 \times 2$ parallel imaging acceleration in pulsatile flow phantom and in vivo with and without respiratory gating. Acta Radiol. 2019; 60:327-37. [PubMed: 30479136]

5. Elbaz MSM, Calkoen EE, Westenberg JJM, et al. Vortex flow during early and late left ventricular filling in normal subjects: quantitative characterization using retrospectively-gated 4D flow cardiovascular magnetic resonance and three-dimensional vortex core analysis. J Cardiovasc Magn Reson. 2014; 16:78. [PubMed: 25270083]

6. Calkoen EE, Elbaz MSM, Westenberg JJM, et al. Altered left ventricular vortex ring formation by 4dimensional flow magnetic resonance imaging after repair of atrioventricular septal defects. J Thorac Cardiovasc Surg. 2015; 150:1233-40. e1 [PubMed: 26282608]

7. Schäfer M, Barker AJ, Kheyfets V, et al. Helicity and vorticity of pulmonary arterial flow in patients with pulmonary hypertension: quantitative analysis of flow formations. J Am Heart Assoc. 2017; 6 e007010 [PubMed: 29263034] 
8. Crandon S, Elbaz MSM, Westenberg JJM, et al. Clinical applications of intra-cardiac fourdimensional flow cardiovascular magnetic resonance: a systematic review. Int J Cardiol. 2017; 249:486-93. [PubMed: 28964555]

9. Crandon S, Westenberg JJM, Swoboda PP, et al. Impact of age and diastolic function on novel, 4D flow CMR biomarkers of left ventricular blood flow kinetic energy. Sci Rep. 2018; 814436 [PubMed: 30258186]

10. Garg P, Swift AJ, Zhong L, et al. Assessment of mitral valve regurgitation by cardiovascular magnetic resonance imaging. Nat Rev Cardiol. 2020; 17:298-312. [PubMed: 31819230]

11. Dyverfeldt P, Bissell M, Barker AJ, et al. 4D flow cardiovascular magnetic resonance consensus statement. J Cardiovasc Magn Reson. 2015; 17:72. [PubMed: 26257141]

12. Feneis JF, Kyubwa E, Atianzar K, et al. 4D flow MRI quantification of mitral and tricuspid regurgitation: reproducibility and consistency relative to conventional MRI. J Magn Reson Imaging. 2018; 48:1147-58. [PubMed: 29638024]

13. Kamphuis VP, Roest AAW, Ajmone Marsan N, et al. Automated cardiac valve tracking for flow quantification with four-dimensional flow MRI. Radiology. 2019; 290:70-8. [PubMed: 30375924]

14. Garg P, Crandon S, Swoboda PP, et al. Left ventricular blood flow kinetic energy after myocardial infarction - insights from 4D flow cardiovascular magnetic resonance. J Cardiovasc Magn Reson. 2018; 20:61. [PubMed: 30165869]

15. Andrews JPM, MacNaught G, Moss AJ, et al. Left ventricular thrombus formation in myocardial infarction is associated with altered left ventricular blood flow energetics [published correction appears in Eur Heart J Cardiovasc Imaging. 2019 Jan 1;20(1):117]. Eur Heart J Cardiovasc Imaging. 2019; 20:108-17. [PubMed: 30137274]

16. Chenevert TL, Brunberg JA, Pipe JG. Anisotropic diffusion in human white matter: demonstration with Mr techniques in vivo. Radiology. 1990; 177:401-5. [PubMed: 2217776]

17. Douek P, Turner R, Pekar J, et al. Mr color mapping of myelin fiber orientation. J ComputAssist Tomogr. 1991; 15:923-9.

18. Beaulieu C. The basis of anisotropic water diffusion in the nervous system - a technical review. NMR Biomed. 2002; 15:435-55. [PubMed: 12489094]

19. Basser PJ. Inferring microstructural features and the physiological state of tissues from diffusionweighted images. NMR Biomed. 1995; 8:333-44. [PubMed: 8739270]

20. Nielles-Vallespin S, Mekkaoui C, Gatehouse P, et al. In vivo diffusion tensor MRI of the human heart: reproducibility of breath-hold and navigator-based approaches [published correction appears in Magn Reson Med. 2014 Aug;72(2):599]. Magn Reson Med. 2013; 70:454-65. [PubMed: 23001828]

21. McGill LA, Ismail TF, Nielles-Vallespin S, et al. Reproducibility of in-vivo diffusion tensor cardiovascular magnetic resonance in hypertrophic cardiomyopathy [published correction appears in J Cardiovasc Magn Reson. 2013;15:22]. J Cardiovasc Magn Reson. 2012; 14:86. [PubMed: 23259835]

22. Tunnicliffe EM, Scott AD, Ferreira P, et al. Intercentre reproducibility of cardiac apparent diffusion coefficient and fractional anisotropy in healthy volunteers. J Cardiovasc Magn Reson. 2014; 16:31. [PubMed: 24886285]

23. McGill LA, Ferreira PF, Scott AD, et al. Relationship between cardiac diffusion tensor imaging parameters and anthropometrics in healthy volunteers. J Cardiovasc Magn Reson. 2016; 18:2. [PubMed: 26738482]

24. Ferreira PF, Kilner PJ, McGill LA, et al. In vivo cardiovascular magnetic resonance diffusion tensor imaging shows evidence of abnormal myocardial laminar orientations and mobility in hypertrophic cardiomyopathy. J Cardiovasc Magn Reson. 2014; 16:87. [PubMed: 25388867]

25. Wu M-T, Tseng W-YI, Su M-YM, et al. Diffusion tensor magnetic resonance imaging mapping the fiber architecture remodeling in human myocardium after infarction: correlation with viability and wall motion. Circulation. 2006; 114:1036-45. [PubMed: 16940196]

26. Winklhofer S, Stoeck CT, Berger N, et al. Post-Mortem cardiac diffusion tensor imaging: detection of myocardial infarction and remodeling of myofiber architecture. Eur Radiol. 2014; 24:2810-8. [PubMed: 25052077] 
27. Mekkaoui C, Jackowski MP, Kostis WJ, et al. Myocardial scar delineation using diffusion tensor magnetic resonance tractography. J Am Heart Assoc. 2018; 7 e007834 [PubMed: 29420216]

28. Ferreira PF, Kilner PJ, McGill L-A, et al. In vivo cardiovascular magnetic resonance diffusion tensor imaging shows evidence of abnormal myocardial laminar orientations and mobility in hypertrophic cardiomyopathy. J Cardiovasc Magn Reson. 2014; 16:87. [PubMed: 25388867]

29. Ariga R, Tunnicliffe EM, Manohar SG, et al. Identification of Myocardial Disarray in Patients With Hypertrophic Cardiomyopathy and Ventricular Arrhythmias. J Am Coll Cardiol. 2019; 73:2493-502. [PubMed: 31118142]

30. Nielles-Vallespin S, Khalique Z, Ferreira PF, et al. Assessment of Myocardial Microstructural Dynamics by In Vivo Diffusion Tensor Cardiac Magnetic Resonance. J Am Coll Cardiol. 2017; 69:661-76. [PubMed: 28183509]

31. von Deuster C, Sammut E, Asner L, et al. Studying dynamic myofiber aggregate reorientation in dilated cardiomyopathy using in vivo magnetic resonance diffusion tensor imaging. Circulation. 2016; 9 e005018 [PubMed: 27729361]

32. Khalique Z, Ferreira PF, Scott AD, et al. Deranged myocyte microstructure in situs inversus totalis demonstrated by diffusion tensor cardiac magnetic resonance. JACC Cardiovasc Imaging. 2018; 11:1360-2. [PubMed: 29361490]

33. Harmer J, Pushparajah K, Toussaint N, et al. In vivo myofibre architecture in the systemic right ventricle. Eur Heart J. 2013; 34:3640. [PubMed: 24142348]

34. Priamo J, Adamopoulos D, Rager O, et al. Downstream indication to revascularization following hybrid cardiac PET/MRI: preliminary results. Nucl Med Commun. 2017; 38:515-22. [PubMed: 28430739]

35. Beitzke D, Rasul S, Lassen ML, et al. Assessment of myocardial viability in ischemic heart disease by PET/MRI: comparison of left ventricular perfusion, hibernation, and scar burden. Acad Radiol. 2020; 27:188-97. [PubMed: 31053482]

36. Bustin A, Ginami G, Cruz G, et al. Five-minute whole-heart coronary MRA with sub-millimeter isotropic resolution, $100 \%$ respiratory scan efficiency, and 3D-PROST reconstruction. Magn Reson Med. 2019; 81:102-15. [PubMed: 30058252]

37. Munoz C, Neji R, Cruz G, et al. Motion-corrected simultaneous cardiac positron emission tomography and coronary MR angiography with high acquisition efficiency. Magn Reson Med. 2018; 79:339-50. [PubMed: 28426162]

38. Robson PM, Dweck MR, Trivieri MG, et al. Coronary artery PET/MR imaging: feasibility, limitations, and solutions. JACC Cardiovasc Imaging. 2017; 10:1103-12. [PubMed: 28109921]

39. Andrews JPM, MacNaught G, Moss AJ, et al. Cardiovascular ${ }^{18} \mathrm{~F}$-fluoride positron emission tomography-magnetic resonance imaging: A comparison study. J Nucl Cardiol. 2019; doi: 10.1007/s12350-019-01962-y

40. Jamar F, Buscombe J, Chiti A, et al. EANM/SNMMI guideline for 18F-FDG use in inflammation and infection. J Nucl Med. 2013; 54:647-58. [PubMed: 23359660]

41. Nensa F, Kloth J, Tezgah E, et al. Feasibility of FDG-PET in myocarditis: comparison to CMR using integrated PET/MRI. J Nucl Cardiol. 2018; 25:785-94. [PubMed: 27638745]

42. Tarkin JM, Joshi FR, Evans NR, et al. Detection of Atherosclerotic Inflammation by ${ }^{68} \mathrm{Ga}-$ DOTATATE PET Compared to [ ${ }^{18}$ F]FDG PET Imaging. J Am Coll Cardiol. 2017; 69:1774-91. [PubMed: 28385306]

43. Writing group; Document reading group; EACVI Reviewers: This document was reviewed by members of the EACVI Scientific Documents Committee for 2014-2016 and 2016-2018. A joint procedural position statement on imaging in cardiac sarcoidosis: from the Cardiovascular and Inflammation \& Infection Committees of the European Association of Nuclear Medicine, the European Association of Cardiovascular Imaging, and the American Society of Nuclear Cardiology. Eur Heart J Cardiovasc Imaging. 2017; 18:1073-89. [PubMed: 28984894]

44. Wisenberg G, Thiessen JD, Pavlovsky W, et al. Same day comparison of PET/CT and PET/MR in patients with cardiac sarcoidosis. J Nucl Cardiol. 2019; doi: 10.1007/s12350-018-01578-8

45. Wicks EC, Menezes LJ, Barnes A, et al. Diagnostic accuracy and prognostic value of simultaneous hybrid 18 F-fluorodeoxyglucose positron emission tomography/magnetic resonance imaging in cardiac sarcoidosis. Eur Heart J Cardiovasc Imaging. 2018; 19:757-67. [PubMed: 29319785] 
46. Dweck MR, Abgral R, Trivieri MG, et al. Hybrid Magnetic Resonance Imaging and Positron Emission Tomography With Fluorodeoxyglucose to Diagnose Active Cardiac Sarcoidosis. JACC Cardiovasc Imaging. 2018; 11:94-107. [PubMed: 28624396]

47. Gillmore JD, Maurer MS, Falk RH, et al. Nonbiopsy diagnosis of cardiac transthyretin amyloidosis. Circulation. 2016; 133:2404-12. [PubMed: 27143678]

48. Trivieri MG, Dweck MR, Abgral R, et al. ${ }^{18}$ F-Sodium Fluoride PET/MR for the Assessment of Cardiac Amyloidosis. J Am Coll Cardiol. 2016; 68:2712-4. [PubMed: 27978955]

49. Dorbala S, Vangala D, Semer J, et al. Imaging cardiac amyloidosis: a pilot study using ${ }^{18} \mathrm{~F}$ florbetapir positron emission tomography. Eur J Nucl Med Mol Imaging. 2014; 41:1652-62. [PubMed: 24841414]

50. Lee S-P, Lee ES, Choi H, et al. 11C-Pittsburgh B PET imaging in cardiac amyloidosis. JACC Cardiovasc Imaging. 2015; 8:50-9. [PubMed: 25499132] 


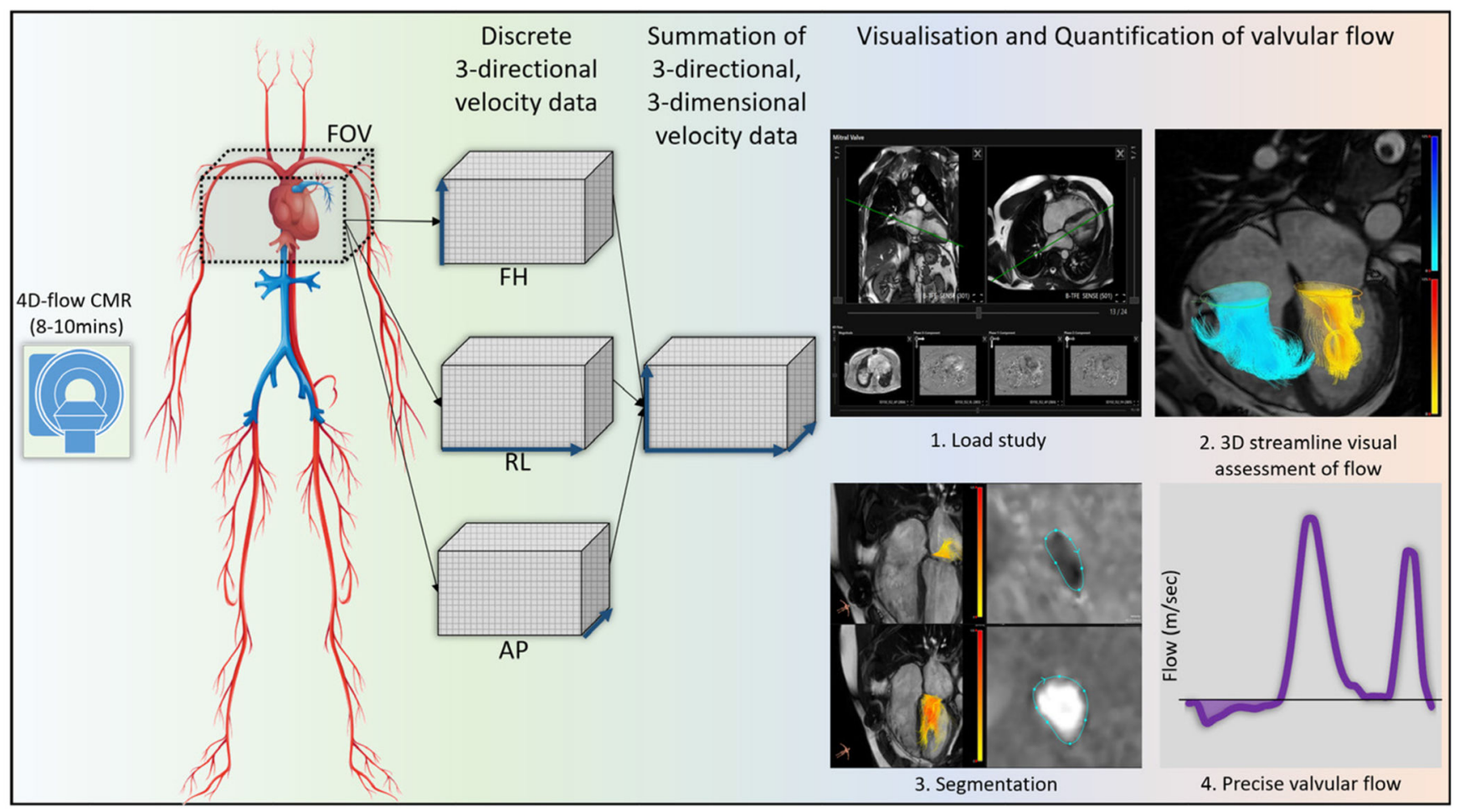

Figure 1.

Overview of four-dimensional (4D)-flow scan. 4D-flow MRI involves acquiring threedirectional velocity data in three-dimension (3D). These data can be then used offline to perform retrospective valve tracking to compute valvular flow. This method factors in valve motion, cardiac motion and allows direct regurgitant jet quantification as demonstrated in steps 3 and 4. 3D, three-dimensional; 4D, four-dimensional;CMR, cardiovascular magnetic resonance; FOV, field-of-view; FH, foot-head; RL, right-left; AP, anterior-posterior. 

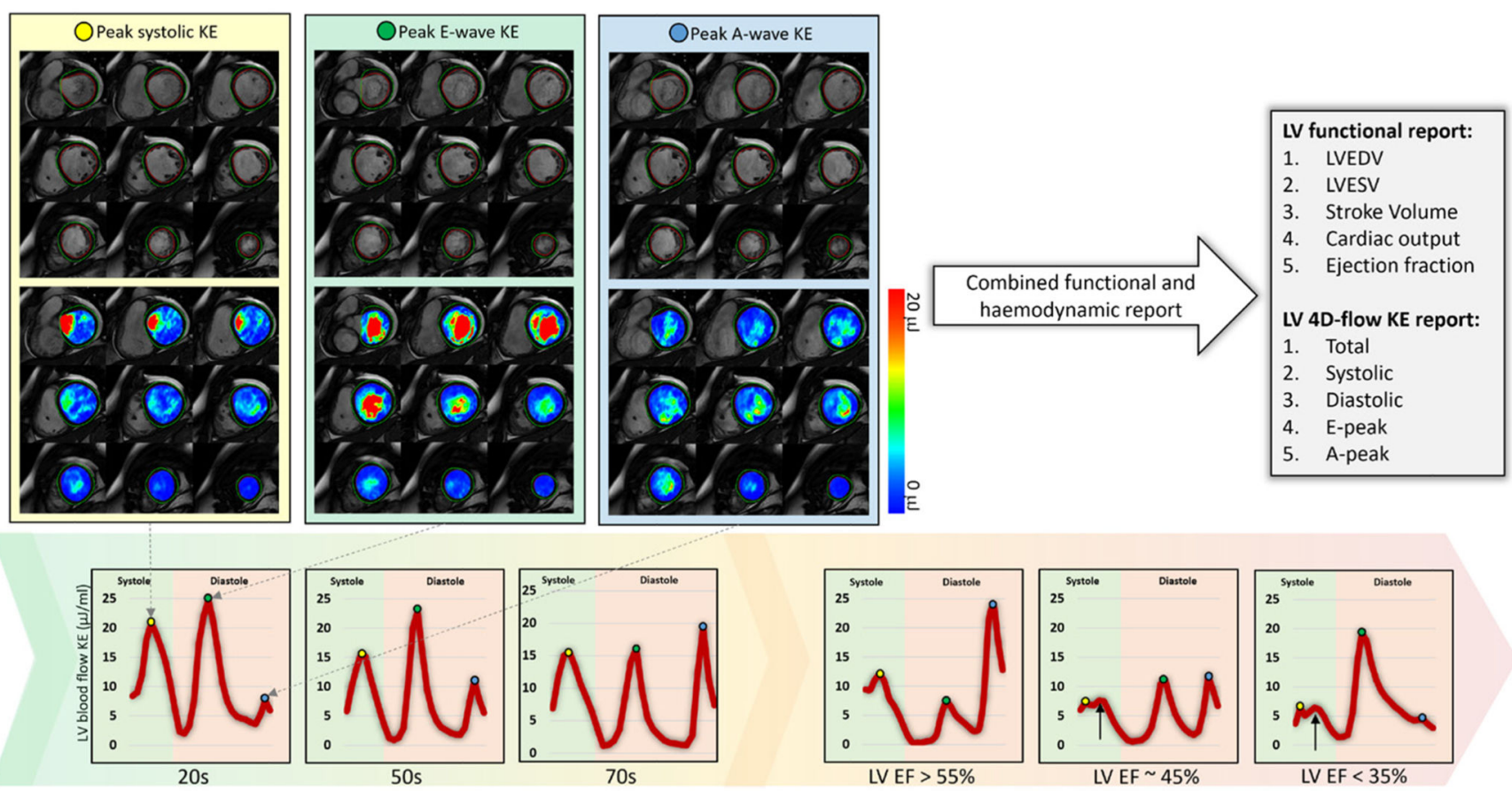

Age-related KE changes
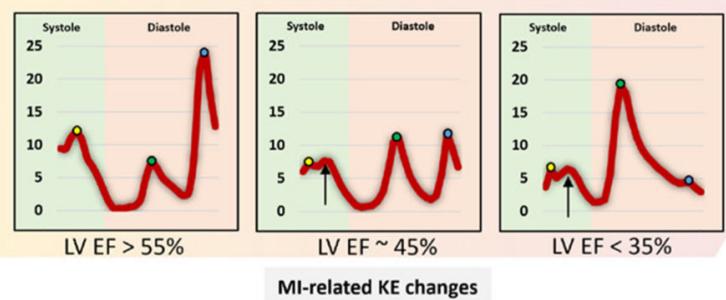

Figure 2.

Four-dimensional (4D) flow cardiovascular magnetic resonance allows reliable and instant quantification of left ventricular blood flow kinetic energy for haemodynamic assessment. Left ventricular (LV) kinetic energy (KE) curves changes with age and sequentially to the degree of LV impairment. This figure demonstrates LV KE maps at three crucial stages of cardiac cycle—peak systole (yellow), peak early filling (green) and late filling (blue). EF, ejection fraction; LVEDV, left ventricular end-diastolic volume; LVESV, left ventricular endsystolic volume; MI, myocardial infarction. 


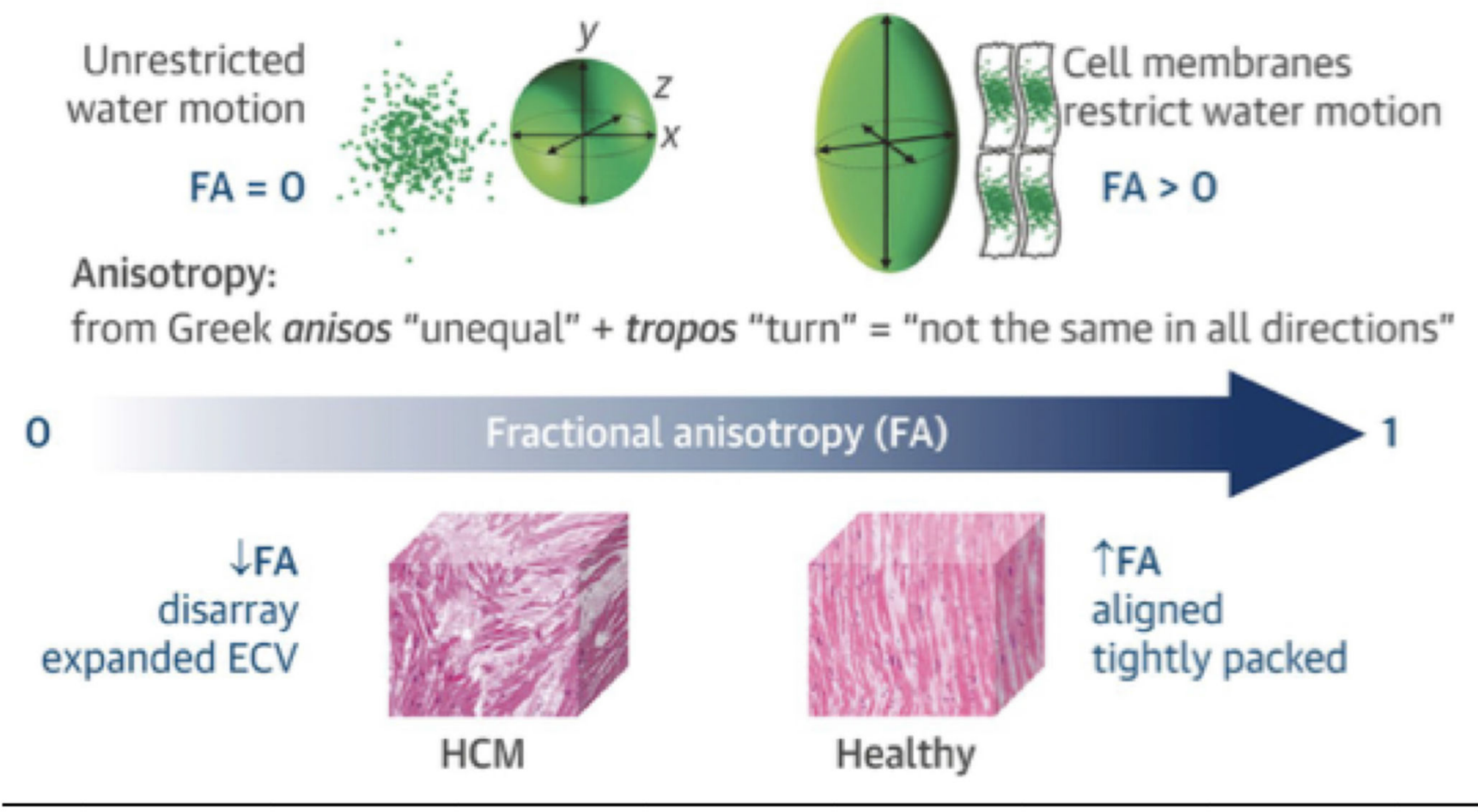

Figure 3.

Illustration of the degree of diffusion restriction in different directions measured using fractional anisotropy (FA). In voxels consisting of coherently aligned myocytes, the FA is expected to be high whereas it is expected be low in voxels with disorganised myocyte orientation such as hypertrophic cardiomyopathy. Reproduced with permission from Tarkin et al. ${ }^{42} \mathrm{ECV}$, extracellular volume; HCM, hypertrophic cardiomyopathy. 


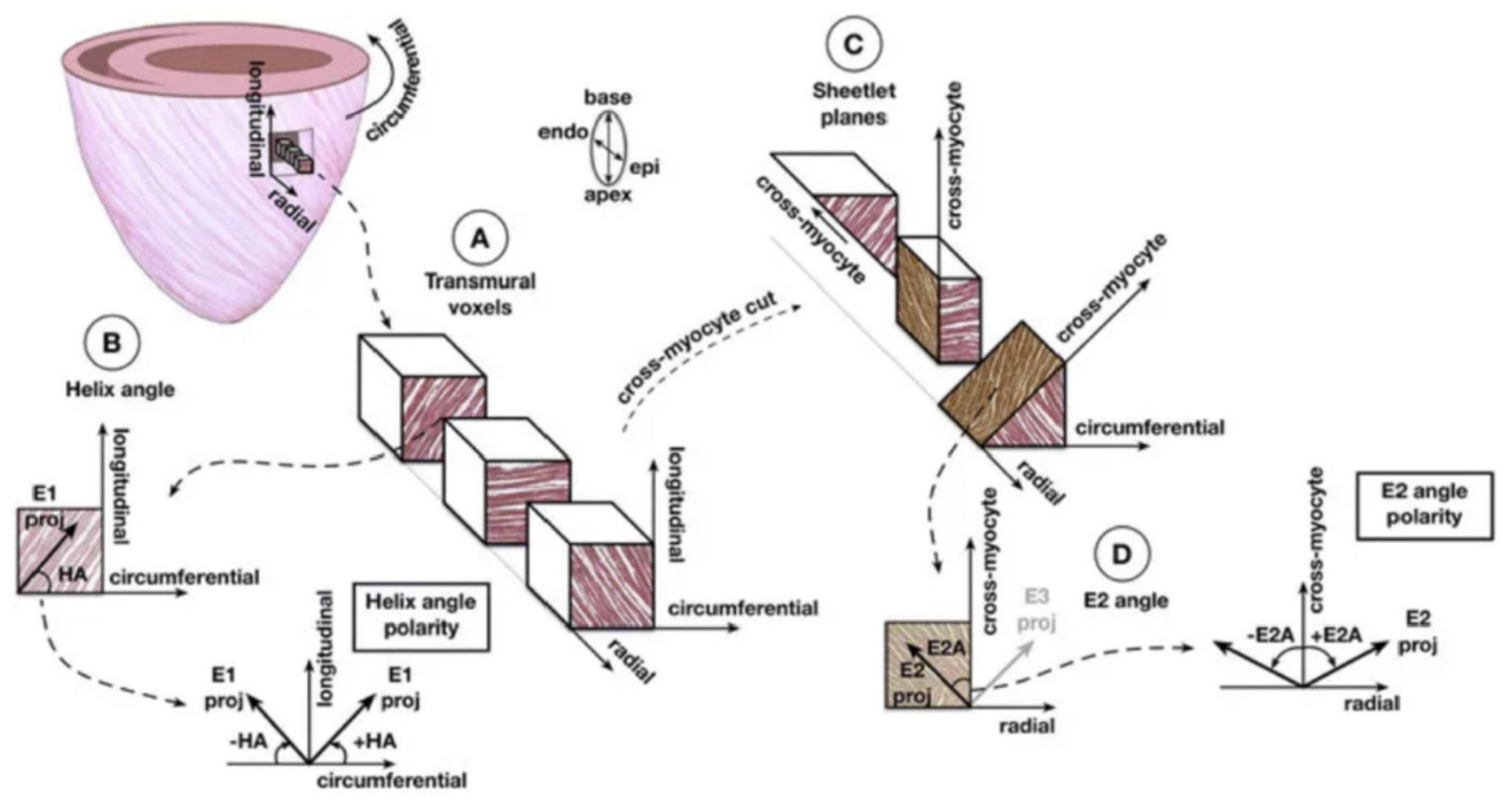

Figure 4.

Diagram illustrating the calculation of helix angle (HA) and second eigenvector angle (E2A)

in each voxel. (A) Illustration of three cubic slices of non-contiguous voxels from the subendocardial, midwall and subepicardial layers. (B) HA is calculated between the circumferential direction and projection of the primary eigenvector. $(C)$ The cubes are sectioned perpendicular to the E1 proj to calculate the cross-myocyte components of diffusion. (D) The E2 angle is measured between the secondary eigenvector projection and cross-myocyte direction in the wall tangent plane. Reproduced with permission from Khalique et al. ${ }^{32}$ 


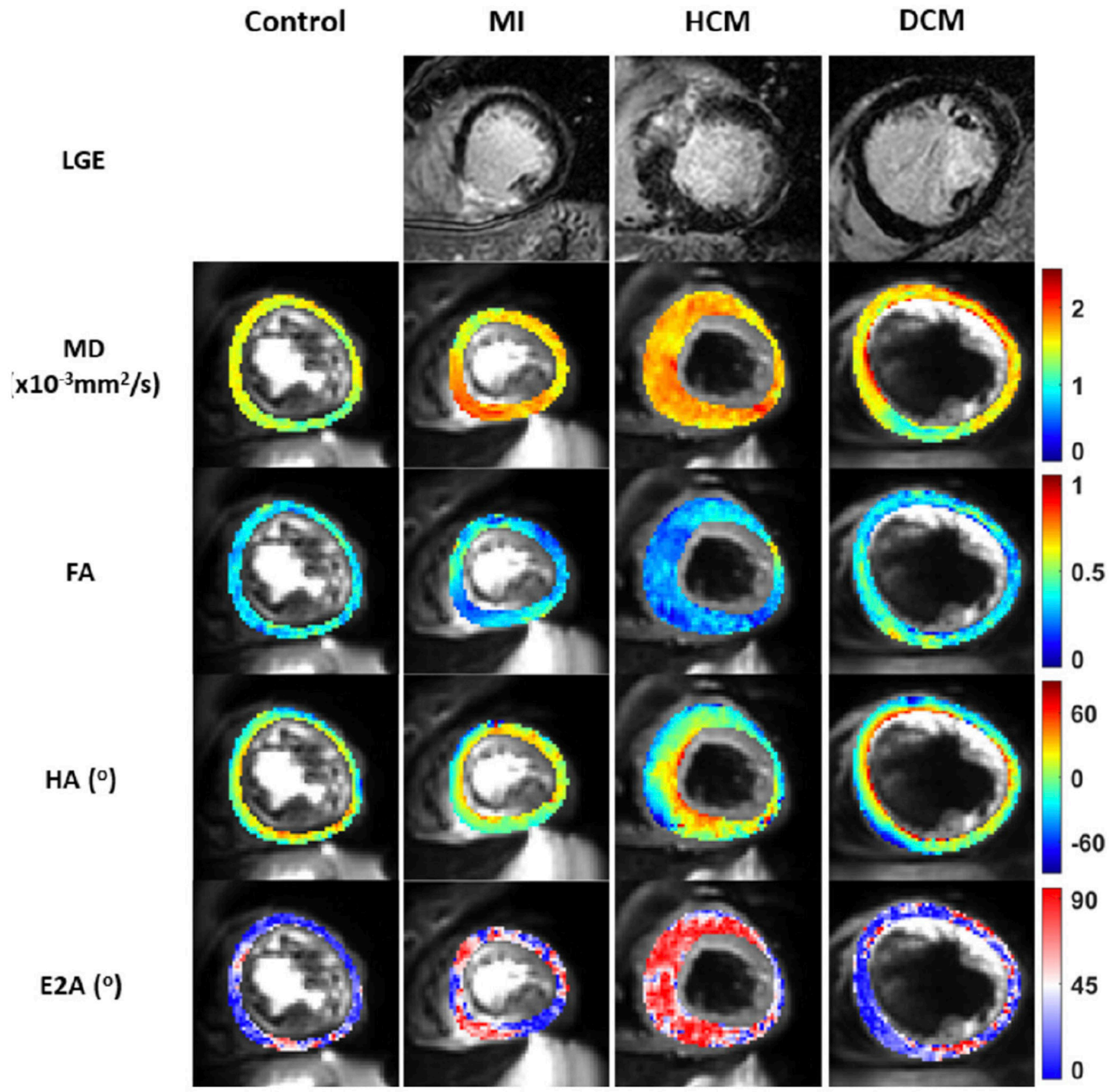

Figure 5.

Multiparametric imaging of the left ventricle myoarchitecture and disarray using diffusion tensor imaging (DTI) and late gadolinium enhancement (LGE). Column A displays a typical basal left ventricular (LV) slice obtained in a healthy control showing a normal mean diffusivity (MD), fractional anisotropy (FA), second eigenvector angle (E2A) and helix angle (HA). Column B demonstrates a basal LV slice in a patient with myocardial infarction (MI) with inferior LGE, raised MD and low FA. Column C demonstrates a basal LV slice in a patient with hypertrophic cardiomyopathy (HCM) with a raised MD and E2A and a low 
FA. Column D demonstrates a basal LV slice in a patient with dilated cardiomyopathy (DCM) demonstrating a raised MD and low FA and E2A. 


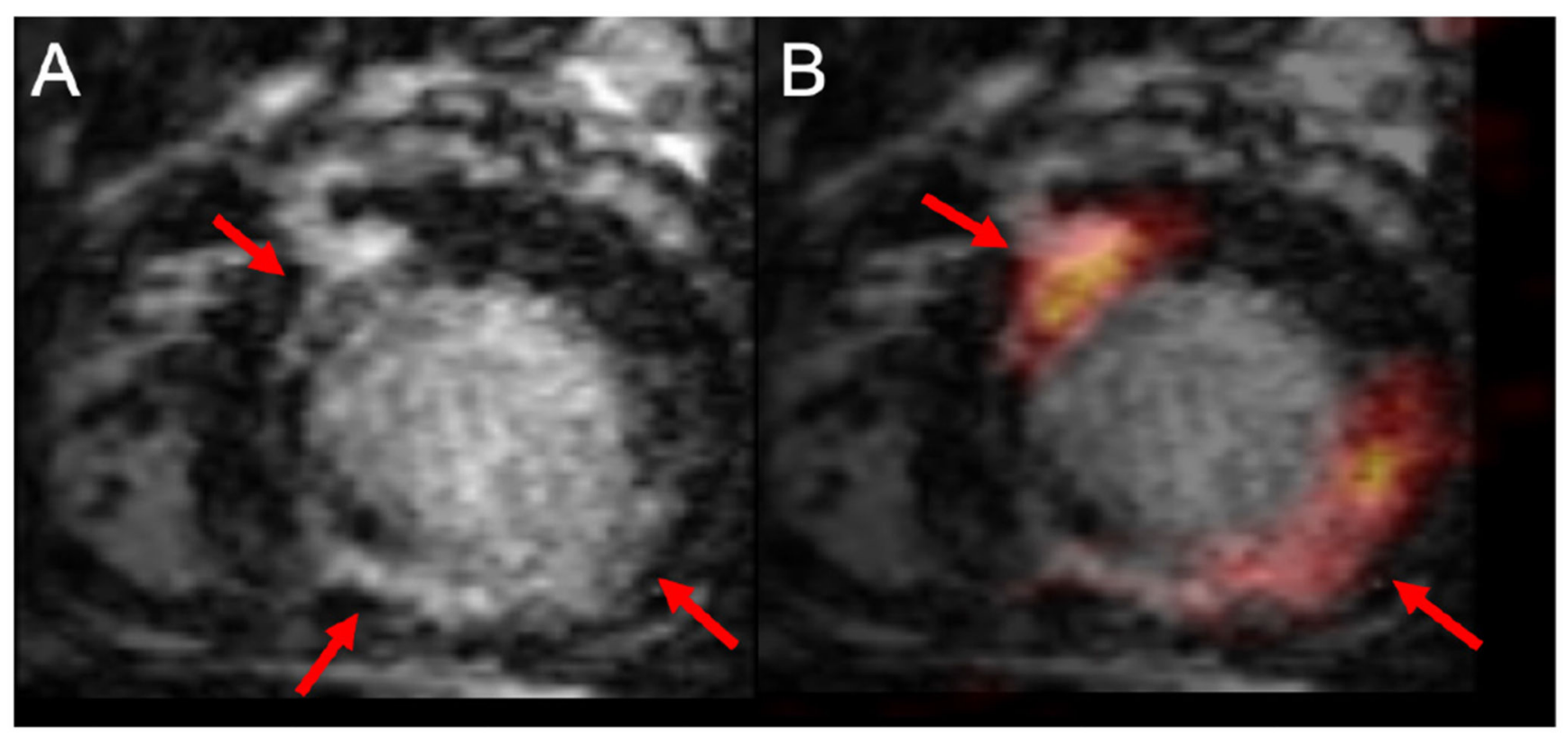

Figure 6.

Positron emission tomography-magnetic resonance (PET-MR) images in a patient with suspected inflammatory heart disease in the short axis orientation. Panel A: reformatted three-dimensional (3D) multiplanar images of isotropic whole heart late gadolinium enhancement (LGE) imaging. There is near circumferential midwall myocardial enhancement of the anteroseptum, inferoseptum, extending to the inferior wall (arrowed). Panel B: image fusion following co-registration of PET and CMR images, demonstrates ${ }^{18} \mathrm{~F}-$ fluorodeoxyglucose myocardial uptake in the anteroseptum and inferolateral wall (arrowed) closely corresponding to midwall LGE. Using hybrid imaging and novel 3D whole heart LGE methods allow for closer registration of data and enhanced motion correction of PET data. An important differential is cardiac sarcoidosis, and hybrid PET-MR may have potential role for diagnosis of patients with cardiac sarcoidosis. Images courtesy of Eliana Reyes, King's College London. 
Table 1

Key principles, clinical translation, advantages, pitfalls and validation studies for 4D-flow, DTI and PET-MR

\begin{tabular}{|c|c|c|c|}
\hline & low & Diffusion Tensor Imaging & PET-MR \\
\hline $\begin{array}{l}\text { Key } \\
\text { principles }\end{array}$ & $\begin{array}{l}\text { Acquires three-directional, three- } \\
\text { dimensional, phase-contrast data } \\
\text { over time (the fourth dimension) } \\
\text { - Allows assessment of } \\
\text { intracardiac flow in three } \\
\text { dimensions }\end{array}$ & $\begin{array}{l}\text { Measures the direction and } \\
\text { magnitude of the diffusion of } \\
\text { water molecules } \\
\text { - In the myocardium, water } \\
\text { diffusion occurs preferentially } \\
\text { along the long axis of } \\
\text { cardiomyocytes } \\
\text { Used to characterise } \\
\text { myocardial microstructure }\end{array}$ & $\begin{array}{l}\text { PET and MRI hardware in the } \\
\text { same gantry allows for } \\
\text { simultaneous PET-MR } \\
\text { acquisition } \\
\text { - Wide potential given } \\
\text { exploration for novel PET } \\
\text { tracers for cardiac application } \\
\text { and emerging MRI methods }\end{array}$ \\
\hline $\begin{array}{l}\text { Clinical } \\
\text { applications }\end{array}$ & $\begin{array}{l}\text { - Insight into complex intracardiac } \\
\text { flow in several disease states } \\
\text { - Quantification of valvular flow- } \\
\text { both forward and backward } \\
\text { (regurgitant) flows, for example, } \\
\text { mitral regurgitation } \\
\text { - Accurate assessment systemic to } \\
\text { pulmonary blood flow } \\
\text { - Visualisation and identification } \\
\text { of shunt in congenital heart } \\
\text { disease } \\
\text { - Identification and assessment of } \\
\text { peak velocity through aortic } \\
\text { valve in three-dimension for } \\
\text { confirming pressure gradient in } \\
\text { aortic stenosis } \\
\text { - Emerging role in left ventricular } \\
\text { diastolic assessment }\end{array}$ & $\begin{array}{l}\text { Myocardial infarction: } \\
\text { depiction of oedema and scar } \\
\text { tissue, changes to the helical } \\
\text { cardiomyocyte arrangement } \\
\text { and myocardial sheetlet } \\
\text { orientations } \\
\text { - Hypertrophic cardiomyopathy: } \\
\text { detect early microstructural } \\
\text { changes and detection of fibre } \\
\text { disarray which can identify } \\
\text { patients at greater risk of } \\
\text { arrhythmias and sudden } \\
\text { cardiac death } \\
\text { Dilated cardiomyopathy: } \\
\text { assessment of myocardial } \\
\text { sheetlet mobility between } \\
\text { diastole and systole, which is } \\
\text { significantly reduced in } \\
\text { patients with DCM } \\
\text { Congenital heart disease: DTI } \\
\text { can help elucidate how } \\
\text { microstructural abnormalities } \\
\text { in impacts on clinical } \\
\text { outcomes }\end{array}$ & $\begin{array}{l}\text { - Evaluation of cardiac masses } \\
\text { using PET and MRI } \\
\text { - Assessment of cardiac } \\
\text { sarcoidosis, although requires } \\
\text { strict dietary preparation. May } \\
\text { help to improve diagnosis and } \\
\text { allow for risk stratification } \\
\text { Emerging role of coronary } \\
\text { imaging with CMRA and PET } \\
\text { imaging of high-risk plaque }\end{array}$ \\
\hline Advantages & $\begin{array}{l}\text { Offline multiplanar assessment of } \\
\text { vascular, valvular and } \\
\text { intracardiac flow } \\
\text { - Allows to quantify transvalvular } \\
\text { flow for all four valves for the } \\
\text { same averaged cardiac cycle- } \\
\text { this improves precision for } \\
\text { Qp:Qs assessment } \\
\text { - Valve tracking allows to improve } \\
\text { valvular flow assessment } \\
\text { - Fast acceleration techniques have } \\
\text { made it possible to acquire 4D- } \\
\text { flow in 6-10 min }\end{array}$ & $\begin{array}{l}\text { - Non-invasive method of } \\
\text { examining in vivo myocardial } \\
\text { microstructure } \\
\text { - Non-contrast sequence } \\
\text { - Can be acquired free-breathing } \\
\text { - Relatively quick acquisition } \\
\text { time }\end{array}$ & $\begin{array}{l}\text { - Simultaneous acquisition } \\
\text { allows precise coregistration of } \\
\text { images and cross-validation of } \\
\text { findings } \\
\text { - PET allows absolute } \\
\text { quantification of biological } \\
\text { processes which can be } \\
\text { combined with } \\
\text { multiparametric data derived } \\
\text { from MRI on anatomy, } \\
\text { morphology and tissue } \\
\text { characterisation } \\
\text { Can use MRI data to motion } \\
\text { correct PET data } \\
\text { - Potential to reduce ionising } \\
\text { radiation dose }\end{array}$ \\
\hline Pitfalls & $\begin{array}{l}\text { Lower temporal resolution when } \\
\text { compared with standard two- } \\
\text { dimensional phase-contrast } \\
\text { imaging }\end{array}$ & $\begin{array}{l}\text { Susceptibility to artefacts from } \\
\text { bulk motion during the cardiac } \\
\text { cycle as well as myocardial } \\
\text { strain during diffusion } \\
\text { encoding, which significantly } \\
\text { impacts on signal-to-noise }\end{array}$ & $\begin{array}{l}\text { Expensive and requires access } \\
\text { to cyclotron or generator for } \\
\text { PET tracers } \\
\text { - Ionising radiation to patients }\end{array}$ \\
\hline
\end{tabular}

ratio 


\begin{tabular}{|c|c|c|c|}
\hline \multicolumn{2}{|c|}{ 4D-flow } & \multirow{2}{*}{$\begin{array}{c}\text { Diffusion Tensor Imaging } \\
\begin{array}{c}\text { Newer sequences require more } \\
\text { powerful gradient hardware }\end{array}\end{array}$} & \multirow{2}{*}{$\begin{array}{l}\text { PET-MR } \\
\begin{array}{l}\text { Challenges with attenuation } \\
\text { correction }\end{array}\end{array}$} \\
\hline & $\begin{array}{l}\text { Cumbersome quality checks and } \\
\text { corrections are necessary for } \\
\text { postprocessing }\end{array}$ & & \\
\hline $\begin{array}{l}\text { Validation } \\
\text { studies }\end{array}$ & $\begin{array}{l}\text { Garg P, Westenberg JJM, van den } \\
\text { Boogaard PJ, Swoboda PP, Aziz } \\
\text { R, Foley JRJ, Fent GJ, Tyl FGJ, } \\
\text { Coratella L, ElBaz MSM, van der } \\
\text { Geest RJ, Higgins DM, } \\
\text { Greenwood JP, Plein S. } \\
\text { Comparison of fast acquisition } \\
\text { strategies in whole-heart four- } \\
\text { dimensional flow cardiac MR: } \\
\text { two-center, 1.5 Tesla, phantom } \\
\text { and in vivo validation study. J } \\
\text { Magn Reson Imaging. 2018 } \\
\text { Jan;47(1):272-281. } \\
\text {-Zhang JM, Tan RS, Zhang S, } \\
\text { Geest RV, Garg P, Leong BR, } \\
\text { Bryant J, Tangcharoen T, Zhao } \\
\text { X, Tan JL, Westenberg JJ, Zhong } \\
\text { L. Comparison of image } \\
\text { acquisition techniques in four- } \\
\text { dimensional flow cardiovascular } \\
\text { mr on } 3 \text { tesla in volunteers and } \\
\text { tetralogy of fallot Patients. Conf } \\
\text { Proc IEEE Eng Med Biol Soc. } \\
\text { 2018 Jul;2018:1115-1118. } \\
\text { - Archer GT, Elhawaz A, Barker } \\
\text { N, Fidock B, Rothman A, van der } \\
\text { Geest RJ, Hose R, Briffa N, Hall } \\
\text { IR, Grech E, Bissell M, Al- } \\
\text { Mohammad A, Treibel TA, Swift } \\
\text { AJ, Wild JM, Garg P. Validation } \\
\text { of four-dimensional flow } \\
\text { cardiovascular magnetic } \\
\text { resonance for aortic stenosis } \\
\text { assessment. Sci Rep. 2020 Jun } \\
\text { 29;10(1):10 569. } \\
\text { - Kamphuis VP, Roest AAW, } \\
\text { Ajmone Marsan N, et al. } \\
\text { Automated cardiac valve tracking } \\
\text { for flow quantification with four- } \\
\text { dimensional flow MRI. } \\
\text { Radiology. 2019 } \\
\text { Jan;290(1):70-78. }\end{array}$ & $\begin{array}{l}\text { Abdullah OM, Drakos SG, } \\
\text { Diakos NA, Wever-Pinzon O, } \\
\text { Kfoury AG, Stehlik J, Selzman } \\
\text { CH, Reid BB, Brunisholz K, } \\
\text { Ratan Verma D, Myrick C, } \\
\text { Sachse FB, Li DY, Hsu EW. } \\
\text { Characterisation Of diffuse } \\
\text { fibrosis in the failing human } \\
\text { Heart Via Diffusion tensor } \\
\text { imaging And quantitative } \\
\text { histological validation nih } \\
\text { public access. NMR Biomed. } \\
\text { 2014;3100(1):1378-86. } \\
\text { Kung GL, Nguyen TC, Itoh A, } \\
\text { Skare S, Ingels NB, Miller DC, } \\
\text { etal. The presence of two local } \\
\text { myocardial sheet populations } \\
\text { confirmed by diffusion tensor } \\
\text { MRI and histological } \\
\text { validation. J Magn Reson } \\
\text { Imaging. 2011 } \\
\text { Teh I, Mcclymont D, Zdora M- } \\
\text { C, Whittington HJ, Davidoiu } \\
\text { V, Lee J, Lygate CA, Rau C, } \\
\text { Zanette I, Schneider JE. } \\
\text { Validation of diffusion tensor } \\
\text { MRI measurements of cardiac } \\
\text { microstructure with structure } \\
\text { tensor synchrotron radiation } \\
\text { imaging. }\end{array}$ & $\begin{array}{l}\text { Nensa F, Tezgah E, Poeppel } \\
\text { TD, Jensen CJ, Schelhorn J, } \\
\text { Kohler J, Heusch P, Bruder O, } \\
\text { Schlosser T, Nassenstein K: } \\
\text { Integrated 18F-FDG PET/MR } \\
\text { imaging in the assessment of } \\
\text { cardiac masses: a pilot study. J } \\
\text { Nucl Med 2015, } \\
\text { 56(2):255-260. } \\
\text { - Dweck MR, Abgral R, Trivieri } \\
\text { MG, Robson PM, Karakatsanis } \\
\text { N, Mani V, Palmisano A, } \\
\text { Miller MA, Lala A, Chang HL } \\
\text { et al: Hybrid MRI and positron } \\
\text { emission tomography with } \\
\text { fluorodeoxyglucose to } \\
\text { diagnose active cardiac } \\
\text { sarcoidosis. JACC Cardiovasc } \\
\text { Imaging 2018, 11(1):94-107. }\end{array}$ \\
\hline
\end{tabular}

4D, four-dimensional; DII, diffusion tensor imaging; PEI-MR, positron emission tomography-magnetic resonance. 